\title{
A proposal of an hybrid meta-strategy for Combinatorial Optimization Problems
}

\author{
Jose M. Framinan * \\ Industrial Management, School of Engineering, University of Seville \\ Rafael Pastor \\ Universidad Politecnica de Catalunya
}

August 16, 2004

\section{Introduction}

In this paper, we propose a procedure for dealing with Combinatorial Optimization Problems (COP). This procedure combines features of exact enumeration methods (i.e. branch and bound) with local search procedures. Regarding the combination of exact and approximate methods for COPs, we are aware of the papers by [5] and [1], but not of similar designs to the one that we present here.

In this paper, we employ as local search procedure the so-called Complete Local Search (CLM) metaheuristic [2]. CLM handles three lists of solutions. The first one, called LIVE, stores solutions that are available for the heuristic for future exploration. A second list, called DEAD, contains solutions that were in LIVE at some stage, and have been already explored. The third list, called NEWGEN is a temporary store for new solutions being generated by the heuristic during the current iteration. CLM starts with a given input solution(s) stored at LIVE, while DEAD and NEWGEN are initially empty. On each iteration of CLM, a solution is picked from LIVE and explored, i.e. all its neighbors are generated and the solution is sent to DEAD. From the set of neighbors, all of them whose objective function is better than a threshold value $\tau$ are checked for membership in LIVE, DEAD and NEWGEN. If not in these lists, the neighbor is put in NEWGEN. At the end of the iteration, all solutions in NEWGEN are transferred to LIVE for the next iteration of CLM. By proper parameter setting, CLM can be transformed into exhaustive search, generic local search with steepest descent or random descent, simulated annealing, or a memory-based metaheuristic in a slightly differ manner than tabu search. Its performance is controlled by a number of parameters such as the threshold value $\tau$, the memory size of the lists, etc. For further details on CLM, the reader is referred to [2]. The remainder of the paper is as follows: first we describe the algorithm and their main features. In section 3 we apply the algorithm to a well-known COP problem: the permutation flow shop scheduling with makespan criterion. Some preliminary computational experience is presented, and section 4 is devoted to some comments and lines for future research.

\section{Description of the algorithm}

The high-level sketch of the proposed algorithm is the following:

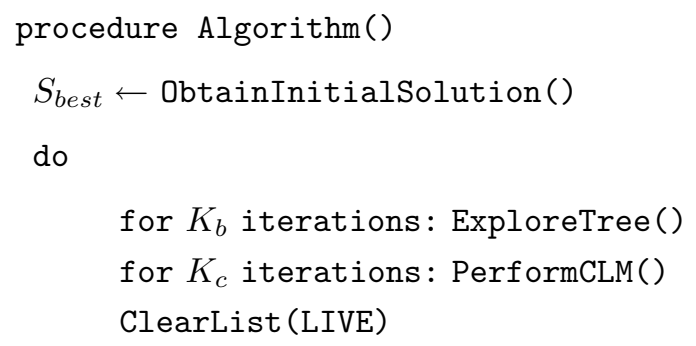

With respect to the procedures employed, procedure ClearList (LIVE) deletes all solutions stored in the list LIVE employed by CLM. Procedure StoppingCondition() establishes the termination criteria of the algorithm. Finally, PerformCLM() performs one iteration of CLM, i.e. takes the first solution in list LIVE, examines all neighbors that have not been explored (they are not in LIVE, DEAD, and NEWGEN), and add those passing a threshold to list LIVE for further exploration. The only difference with respect to the original CLM is that in our procedure, neighbors are checked not to belong to list FORBIDDEN (discussed in next subsection). Finally, ExploreTree() is described in the next subsection.

*Present address: Escuela Superior de Ingenieros, Camino de los Descubrimientos, s/n E 41092 Seville, Spain. Email: jose@esi.us.es 


\subsection{Procedure ExploreTree()}

An iteration of ExploreTree() opens a promising node (constituted by a partial solution stored in a list TREE) into a number of nodes of the next level. A lower bound for the nodes is calculated in order to branch the fathomed nodes, who are included in the list FORBIDDEN. The purpose is to prevent CLM to explore solutions that are 'children' of these nodes, as mentioned before. Among the non fathomed nodes, the most promising one is selected so it can guide the CLM procedure to a region where good solutions can be found (this is done by employing a weighting function that will be discussed later). As the CLM procedure requires a complete solution, a complete solution should be generated from the partial solution represented by this specific node (this procedure is discussed later in subsection 2.1.3). Once a complete solution is generated, it is added to LIVE so it can be explored by the CLM algorithm. In detail, the algorithm operates as follows:

procedure ExploreTree()

begin

$S \leftarrow$ GetNodeFromTree()

delete selected node from list TREE

if adding a component $c$ to $S$ produces a complete solution:

$S_{c} \leftarrow$ by adding solution component $c$ to $S$.

if $U B\left(S_{c}\right)<f\left(S_{\text {best }}\right)$, then $S_{\text {best }} \leftarrow S_{c}$

add $S_{c}$ to LIVE

else

for all components $i$ that can be appended to the partial solution $S$ :

$S_{i} \leftarrow$ by adding solution component $i$ to $S$.

$L B\left(S_{i}\right) \leftarrow$ CalculateLowerBound $\left(S_{i}\right)$.

if $L B\left(S_{i}\right)<f\left(S_{\text {best }}\right)$

add $S_{i}$ to TREE.

else

add $S_{i}$ to FORBIDDEN.

end

end

select $S_{r}:=\left\{S_{r}: L B\left(S_{r}\right) \leq L B\left(S_{i}\right)\right\}$.

$S \leftarrow$ ObtainCompleteSolFromNode $\left(S_{r}\right)$.

if $f(S)<f\left(S_{\text {best }}\right)$, then $S_{\text {best }} \leftarrow S$

add $S$ to LIVE

end

if $S_{\text {best }}$ has been improved, then UpdateTree()

end of procedure ExploreTree()

Basically, the procedure ExploreTree() selects one node from the list TREE according to a weighting function -if the nodes in TREE are sorted according to this weighting function, then it simply picks the first node from the list. If adding a new component to the node makes a complete solution (the maximum level in the depth of the branch and bound has been reached), then this solution is compared to the best-so-far solution. If it is better, then a new bestso-far solution is found. The solution is added to LIVE as a solution whose neighborhood is worth to be investigated by the CLM procedure. In case that adding a new component to the node does not make a complete solution, then a good complete solution is obtained by making use of procedure ObtainCompleteSolFromNode(), which is explained in section 2.1.3.

\subsubsection{Procedure GetNodeFromTree()}

This procedure basically picks a node from the node list in TREE. If the nodes in TREE are sorted in ascending order of the weighting function, then this procedure takes the most promising node of TREE regardless the length of the partial solution (or equivalently, the level of the node in the branch and bound terminology). In contrast, if the nodes in TREE are sorted according to their level (length of the partial solution) and ties are broken according to the weighting function, then the procedure performs a 'first depth search' in the node tree.

If, when invoking this procedure, there is no more nodes in TREE, then the algorithm stops as the optimal solution has been found.

Finally, with respect to the weighting function, it is clear that it has a crucial role in the performance of the algorithm. Some judicious choices for the weighting function include:

- The lower bound $(L B)$ of the node.

- The average between the lower bound and an upper bound $(U B)$ of the node, i.e. $(U B+L B) / 2$ 


\subsubsection{Procedure UpdateTree $(S)$}

This procedure deletes from list TREE all nodes whose lower bound is greater than $C_{\max }(S)$. This procedure is invoked whenever the current best solution is improved. Or, in the branch and bound terminology, whenever a new upper bound is found.

\subsubsection{Procedure ObtainCompleteSolFromNode $(S)$}

There are several sensible ways to obtain a complete solution from the partial solution represented by the most promising node. These include:

- LIVE based- procedure. This procedure consists in searching in list LIVE for complete solutions in the vertex of this node and place them as next solutions to be explored by CLM. When using this option, it should be taken into account the possibility that no solution in LIVE corresponds to the node selected.

- TREE based- procedure. In this option, a complete solution solely based in the list TREE should be obtained. Therefore, the node selected should be expanded into child nodes until a complete solution is obtained.

- Heuristic based- procedure. This procedure consists in completing the partial solution by means of some heuristic. A sensible way to do it is using ad-hoc constructive heuristics for the problem under consideration in order to obtain a complete solution.

This third option is selected for the implementation of the algorithm to the $F|p r m u| C_{\max }$ problem and will be discussed in section 3 , as it is problem-specific.

\section{Implementation: the $F \mid$ prmu $\mid C_{\max }$ problem}

In order to test the proposed approach, we have implemented a version of the algorithm for the $F \mid$ prmu $\mid C_{\max }$ problem. This problem is a well-known combinatorial optimization problem, and we refer the reader to references [3] and [4] for the latest advances on this problem. In our implementation of the proposed algorithm, the following decisions have been adopted:

- The solutions for the problem are coded employing the most common codification, i.e. a solution for a $n$-jobs, $m$-machines problem is defined by a vector $S=\left(\sigma_{1}, \ldots, \sigma_{n}\right)$ where $\sigma_{i}$ denotes the job being sequenced in order $i$ th. An insertion-based neighborhood has been defined for CLM, consisting in removing a job from position $i$ and inserting it into position $j$. A node of level $k$ th is represented by a partial sequence where the first $k$ jobs are already fixed. The set of components $i$ that can be appended to a partial solution (node) is constituted by the set of non-scheduled jobs.

- As procedure ObtainCompleteSolFromNode(), a heuristic-based procedure has been designed. This procedure is based on the NEH heuristic [6], which is considered the best constructive heuristic for the problem under consideration. According to the NEH heuristic, jobs are arranged in descending order of the sum of their processing times. Then, each job $k$ th is inserted on each of the $(k+1)$ th possible slots of the partial sequence, and the so-obtained partial sequence yielding the lowest (partial) makespan is retained as partial sequence for the next step. In our implementation of ObtainCompleteSolFromNode(), already $k$ jobs have been scheduled (in the first $k$ th positions). Therefore, the same steps in the NEH heuristic are applied for the non-scheduled jobs, i.e. they are arranged in descending order of the sum of their processing times, and then job $i$ th is inserted in positions from $(k+1)$ to $(i+1)$. The best partial sequence is retained for the next step.

- The selection of the next node to be processed -procedure GetNodeFromTree()-is done such as nodes in TREE are sorted according to their lower bounds regardless the length of the partial schedule (level of the node). Thus the weighting function employed is a lower bound of the solution. In this implementation, a fast, simple, lower bound for the makespan that can be computed in $O(n m)$ has been selected. This lower bound is denoted as $L B 1$ in the review by $\underline{[4]}$.

- Parameter setting of the algorithm:

- $K_{b}$ has been set to 10. As one new solution is added to LIVE on each iteration of ExploreTree(), this means that 10 solutions are stored in LIVE for CLM.

- $K_{c}$ has been set to $K_{c}=K_{b} \cdot|\mathrm{LIVE}|$. This ensures that all relevant solutions in LIVE are to be explored before LIVE is cleared.

- As stopping criterion, it has been set to stop after 100 iterations without improvement. This number of iterations have been proved to be more than sufficient to obtain accurate solutions of the problem. In view of the results shown in table 1 , we conjecture that a similar performance could have been obtained with a lower number of iterations. 


\begin{tabular}{|c|c|c|c|c|c|c|}
\hline Problem & \multicolumn{2}{|c|}{ Size } & \multicolumn{2}{|c|}{ Makespan } & \multicolumn{2}{|c|}{ PRD } \\
\hline Instance & $n$ & $m$ & $U B_{T}$ & $C_{\max }(A L G)$ & Indiv. & Avg. \\
\hline ta001 & 20 & 5 & 1278 & 1278 & 0.000 & \\
\hline ta002 & 20 & 5 & 1359 & 1359 & 0.000 & \\
\hline ta003 & 20 & 5 & 1081 & 1081 & 0.000 & \\
\hline ta004 & 20 & 5 & 1293 & 1293 & 0.000 & \\
\hline ta005 & 20 & 5 & 1235 & 1235 & 0.000 & \\
\hline ta006 & 20 & 5 & 1195 & 1195 & 0.000 & \\
\hline ta007 & 20 & 5 & 1234 & 1239 & 0.405 & \\
\hline ta008 & 20 & 5 & 1206 & 1206 & 0.000 & \\
\hline ta009 & 20 & 5 & 1230 & 1240 & 0.813 & \\
\hline ta010 & 20 & 5 & 1108 & 1108 & 0.000 & 0.122 \\
\hline ta011 & 20 & 10 & 1582 & 1582 & 0.000 & \\
\hline ta012 & 20 & 10 & 1659 & 1659 & 0.000 & \\
\hline ta013 & 20 & 10 & 1496 & 1496 & 0.000 & \\
\hline ta014 & 20 & 10 & 1377 & 1379 & 0.145 & \\
\hline ta015 & 20 & 10 & 1419 & 1419 & 0.000 & \\
\hline ta016 & 20 & 10 & 1397 & 1397 & 0.000 & \\
\hline ta017 & 20 & 10 & 1484 & 1484 & 0.000 & \\
\hline $\operatorname{ta} 018$ & 20 & 10 & 1538 & 1543 & 0.325 & \\
\hline ta019 & 20 & 10 & 1593 & 1593 & 0.000 & \\
\hline ta020 & 20 & 10 & 1591 & 1591 & 0.000 & 0.047 \\
\hline ta021 & 20 & 20 & 2297 & 2298 & 0.044 & \\
\hline ta022 & 20 & 20 & 2099 & 2099 & 0.000 & \\
\hline $\operatorname{ta} 023$ & 20 & 20 & 2326 & 2326 & 0.000 & \\
\hline ta024 & 20 & 20 & 2223 & 2223 & 0.000 & \\
\hline ta025 & 20 & 20 & 2291 & 2296 & 0.218 & \\
\hline ta026 & 20 & 20 & 2226 & 2230 & 0.180 & \\
\hline ta027 & 20 & 20 & 2273 & 2276 & 0.132 & \\
\hline ta028 & 20 & 20 & 2200 & 2200 & 0.000 & \\
\hline ta029 & 20 & 20 & 2237 & 2237 & 0.000 & \\
\hline ta030 & 20 & 20 & 2178 & 2178 & 0.000 & 0.057 \\
\hline ta031 & 50 & 5 & 2724 & 2724 & 0.000 & \\
\hline ta032 & 50 & 5 & 2834 & 2834 & 0.000 & \\
\hline ta033 & 50 & 5 & 2621 & 2621 & 0.000 & \\
\hline ta034 & 50 & 5 & 2751 & 2751 & 0.000 & \\
\hline ta035 & 50 & 5 & 2863 & 2863 & 0.000 & \\
\hline ta036 & 50 & 5 & 2829 & 2829 & 0.000 & \\
\hline ta037 & 50 & 5 & 2725 & 2725 & 0.000 & \\
\hline ta038 & 50 & 5 & 2683 & 2683 & 0.000 & \\
\hline ta039 & 50 & 5 & 2552 & 2561 & 0.353 & \\
\hline ta040 & 50 & 5 & 2782 & 2782 & 0.000 & 0.035 \\
\hline Average & & & & & 0.065 & \\
\hline
\end{tabular}

Table 1: Results obtained in a subset of the testbed by $\underline{[9]}$

- Regarding CLM parameters, $K$ has been set to 1 , and $\alpha_{0}$ and $\beta$ have set set to zero. This implies that the threshold is the makespan of the current solution, that is: the procedure includes in LIVE these solutions whose makespan improves the makespan obtained for the current solution (see $\underline{[2\rceil}$ for details on the performance of the different parameters for CLM).

Note that we have not employed in our implementation neither sophisticated (and accurate) lower bounds such as the ones described in [4], nor problem-specific neighborhoods, such as those described in [3]. On one hand, such lower bounds are computationally very expensive and their inclusion might distort the balance between the branch and bound and the local search algorithm. On the other hand, using tailored neighborhoods might hide the general capabilities of the algorithm. However, we believe that the current results can be improved by the introduction of these two elements, being this an interesting area of future research (see section 4 with respect to this issue).

To test the performance of the implementation, part of the testbed of problem instances built by Taillard [9] has been selected. The best-so-far results obtained for these instances are available in the OR Library [7] and are denoted in this paper as $U B_{T}$. For each instance, $C_{\max }(A L G)$ the makespan obtained by the implementation has been measured in terms of the Percentage Relative Difference(PRD), defined as follows:

$$
P R D=\frac{C_{\max }(A L G)-U B_{T}}{U B_{T}} \cdot 100
$$

The results of the experiments are shown in table 1. The quality of the solutions is extremely good, reaching the best known solution in 31 out of the 40 instances. Regarding the computation times, they grow rather fast with the problem size.

\section{Comments and final remarks}

In this paper we present the outline of a hybrid metaheuristic for COPs. The algorithm combines features of exact enumeration methods (i.e. branch and bound with some features borrowed from beam search) with local search strategies. The communication between local search and enumeration procedure is ensured so both algorithms efficiently cooperate. On one hand, the local search provides the enumeration procedure with accurate lower bounds. On the 
other hand, the enumeration procedure drives the local search by guiding the latter to new regions where good solutions can be found and providing a diversification mechanism for avoiding local optima. Finally, it has to be noted that the procedure yields exact -optimal-solutions if allowed sufficient computation time.

This procedure has been implemented for a well-known COP, the the $F \mid$ prmu $\mid C_{\text {max }}$ problem. The preliminary results are encouraging, despite the implementation does not use tailored neighborhoods nor sophisticated lower bounds.

At this stage of the research, our effort concentrates in the following lines:

- Additional features from tree search methods (particularly beam-search [8]) can be incorporated, including the use of a beam width to reduce the computational burden. However, these new features would imply the loss of guarantee of optimality.

- Different local search strategies can be incorporated instead of CLM. The most obvious choice is tabu search, which is also expected to reduce the computational burden of the local search part as it does not need to handle a list of all explored solutions.

- The procedure should be applied to different COPs. We believe that this could be particularly fruitful in its application to problems with severe feasibility restrictions (i.e. problems where their natural codification contemplates unfeasible solutions).

- Regarding the specific implementation that has been presented in this paper, a lot of work has yet to be done. On one hand, it has been already mentioned that using specific (reduced) neighborhoods and accurate lower bound is expected to improve the algorithm. On the other hand, a fine tuning of the parameters and decisions involved in the algorithm should be carried out in order to increase its performance.

\section{References}

[1] French, A.P., Robinson, A.C., Wilson, J.M., (2001) Using a hybird genetic-algorithm/branch and bound approach to solve feasibility and optimization integer programming problems, Journal of Heuristics, 7, 551-564.

[2] Ghosh, D. and Sierksma, G. (2002) Complete Local Search with Memory, Journal of Heuristics, 8, 571-584.

[3] Grabowski, J., Wodecki, M. (2004) A very fast tabu search algorithm for the permutation flow shop problem with makespan criterion, Computers \& Operations Research, 31, 1891-1909.

[4] Ladhari, T., Haourari, M. (2004) A computational study of the permutation flow shop problem based on a tight lower bound, Computers $\mathscr{E}$ Operations Research, In press, available online.

[5] Nagar, A., Heragu S.S., Haddock, J. (1996) A combined branch and bound and genetic algorithm based for a flowshop scheduling algorithm, Annals of Operations Research, 63, 397-414.

[6] Nawaz, M., Enscore, E.E., Ham, I. (1983) A heuristic algorithm for the $n$-job, $m$-machine flow shop sequencing problem, Omega, 11, 91-95.

[7] OR Library: http://mscmga.ms.ic.ac.uk/info.html.

[8] Ow, P.S., Morton, T.E. (1988) Filtered beam search in scheduling, International Journal of Production Research, 26, 35-62.

[9] Taillard, E. (1993) Benchmark for basic scheduling problems, European Journal of Operational Research, 64, 278-285. 